\title{
Study on the performance of journal bearings in different lubricants by CFD and FSI method with thermal effect and cavitation
}

\author{
Hulin $\mathrm{Li}^{1}$, Yanzhen Wang ${ }^{1}$, Ning Zhong ${ }^{2}$, Yonghong Chen ${ }^{2}$ and Zhongwei Yin ${ }^{1}$ \\ ${ }^{1}$ School of Mechanical Engineering, Shanghai Jiao Tong University, 800 Dongchuan RD, Minhang District, Shanghai 200240, PR China \\ ${ }^{2}$ Chongqing Yuejin Machinery Co., Ltd., No.1 Huagong RD, Yongchuan District, Chongqing 402169, PR China
}

\begin{abstract}
This paper used a new transient computational fluid dynamics and fluid-structure interaction method to investigate the journal bearing performance with the effect of thermal and cavitation, to reveal the performance of journal bearing in different lubricants and to provide substitution references for bearings in different lubricants. Considering thermal effect, elastic deformation and cavitation, a detailed discussion was conducted to show the performance of plain journal bearings lubricated by water, seawater, and lubricating oil by computational fluid dynamics (CFD) and fluid structure interaction (FSI) method. And the results in this work are compared with the published results. The variation of dimensionless load carrying capacity, maximum film pressure and temperature with eccentricity ratio are presented, which can provide reference for the design of bearings. Furthermore, a diagram is presented for journal bearings with different diameter, length-diameter ratio and lubricants, which can be used as a reference for the equivalent substitutions of bearings. The present research provides references as to the design of bearings and the substitutions of bearings by different lubricants.
\end{abstract}

\section{Introduction}

Water-lubricated journal bearings are drawing increasing attention due to the continuous ecological concern. They are used in shipbuilding and industrial equipment for the advantages of environmental friendliness, low cost, and low friction coefficient $[1,2]$. It is a new tendency that oil-lubricated bearings are being replaced by water-lubricated ones $[3,4]$. Due to the fact that physical parameters have great differences among water, seawater and lubricating oil, the lubrication performances such as load carrying capacity and temperature rise of water or seawater-lubricated bearings are much more different than those of oil lubricated bearings. Therefore, it is necessary to carry out relevant studies, so as to provide theoretical basis for the replacement of oil lubrication by water lubrication.

The researches for water-lubricated journal bearing is mainly about tribological behavior of bearing material[5, 6] and experimental researches of bearing performance[7]-[9]. In our previous work [3], the corresponding relationship of diameter of oil-lubricated and water-lubricated journal bearings was analyzed at a constant load of $3920 \mathrm{~N}$. However, the influence of elastic deformation, eccentricity ratio, different bearing diameters and length-diameter ratio were not considered in previous work. Therefore, the present study aims to investigate the lubrication performance of water-lubricated and oil-lubricated journal bearings including elastic deformation, thermal effect and cavitation and to provide references for the replacement of oil lubrication by water lubrication. Furthermore, the effects of eccentricity ratio, diameters and length to diameter ratios on lubrication performance are considered The lubricants include water, seawater, and lubricating oil. Finally, the equivalent substitutes of bearings among different lubricants and diameters are discussed, which is a pivotal judgment for the replacement of oil lubricated bearings by water or seawater ones.

\section{Theoretical}

\subsection{Governing equations of the fluid}

Conservation equations are used to predict the performance of fluid domain.

Mass conservation equation

$$
\frac{\partial \rho_{f}}{\partial \mathrm{t}}+\nabla \cdot(\rho \overrightarrow{\mathbf{v}})=0
$$

where $\rho_{f}$ is the fluid density, and $\mathbf{v}$ is the fluid velocity vector.

Momentum conservation equations

$$
\frac{\partial}{\partial \mathrm{t}}\left(\rho_{f} \mathbf{v}\right)+\nabla \cdot\left(\rho_{f} \mathbf{v v}\right)=-\nabla \mathrm{P}+\nabla \cdot\left(\tau_{f}\right)+\rho_{f} \boldsymbol{g}+\boldsymbol{F}
$$

where $\mathrm{P}$ is the static pressure, $\tau_{f}$ is the stress tensor, $\rho_{f} g$ 
isthe gravitational body force, and $\boldsymbol{F}$ is the external body force. The stress tensor $\tau_{f}$ is defined as

$$
\tau_{\mathrm{f}}=\mu_{f}\left[\left(\nabla \mathbf{v}+\nabla \mathbf{v}^{T}\right)-2 / 3 \nabla \times \mathbf{v} I\right]
$$

where $\mu_{\mathrm{f}}$ is the fluid viscosity and $\mathrm{I}$ is the unit tensor. Energy conservation equation

$$
\rho_{f} \frac{\partial}{\partial t}\left(\boldsymbol{e}+\frac{v^{2}}{2}\right)=\rho_{f} \boldsymbol{F} \cdot \boldsymbol{v}+\nabla \cdot(P \boldsymbol{v})+\nabla \cdot(k \nabla T)
$$

where $e$ is the internal energy of fluid, $k$ is the coefficient of thermal conductivity, and $T$ is the temperature of fluid.

\subsection{Fluid-structure interaction}

The nodes on the fluid-solid interface conform to the displacement compatibility, traction equilibrium, and thermal equilibrium equations.

$$
\left\{\begin{aligned}
\mathbf{d}_{f} & =\mathbf{d}_{s} \\
\mathbf{n} \cdot \tau_{f} & =\mathbf{n} \cdot \tau_{s} \\
\mathrm{~T}_{\mathrm{f}} & =\mathrm{T}_{s}
\end{aligned}\right.
$$

where $\boldsymbol{d}_{\boldsymbol{f}}$ and $\boldsymbol{d}_{\boldsymbol{s}}$ are the displacements of fluid domain and structure respectively. $\tau_{f}$ and $\tau_{\mathrm{s}}$ are the stress of fluid domain and structure respectively. $\mathrm{T}_{\mathrm{f}}$ is the temperature of fluid, and $\mathrm{T}_{\mathrm{s}}$ is the temperature of solid.

\section{Models}

The schematics of the journal and bearing system are shown in Fig.1, and the corresponding parameters needed in calculation are listed in Table 1. Only the bearing model was created for the shaft was assumed to be rigid. The boundary conditions of outside surface of the bush were used as fixed support, the inside surface fluid solid interface, as shown in Fig. 2 b. The time step was set to $0.001 \mathrm{~s}$.

The numerical results were calculated by ANSYS CFX. The rotational speed was $2000 \mathrm{r} / \mathrm{min}$ in the current study. The two end faces of the fluid domain were taken as inlet and outlet with gauge pressure being zero Pascal. The inner face of fluid domain was modeled as rotational wall and the outer face stationary wall, as shown in Fig.2 a. Cavitation was modeled by Rayleigh-Plesset model for its stability and liable to convergence. The heat transfer model was selected as fluid dependent because of its fitness for multiphase. The cavitation pressure of water lubrication pressure was assumed to be $2340 \mathrm{~Pa}$. The cavitation pressure of lubricating oil was set to $0 \mathrm{~Pa}$ for the cavitation of oil-lubricated bearing was mostly caused by the escape of dissolved air. The bearing material was steel, and the lubricants were water, seawater, and lubricating oil.
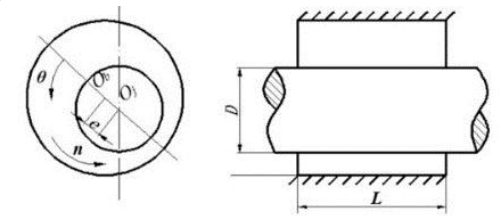

Figure 1. Structure schematic diagram of journal bearing

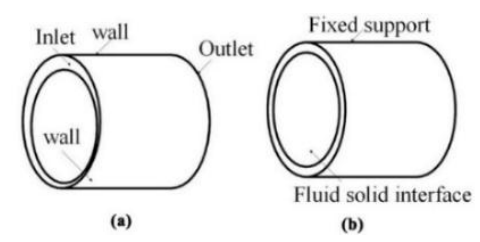

Figure 2. Boundary conditions

Table 1 Parameters of the models

\begin{tabular}{|l|l|}
\hline Bearing diameter & $80 \mathrm{~mm}$ \\
\hline Bushing thickness & $5 \mathrm{~mm}$ \\
\hline Radial clearance & $0.04 \mathrm{~mm}$ \\
\hline Steel Young's modulus & $115 \mathrm{GPa}$ \\
\hline Steel Poisson's ratio & 0.33 \\
\hline
\end{tabular}

\section{Results and discussion}

\subsection{Influence of thermal effect and elastic deformation}

The effect of thermal and elastic deformation on bearing performance are studied with the parameter of $\mathrm{D}=80 \mathrm{~mm}$, $\mathrm{L} / \mathrm{D}=1, \Phi=0.1 \%, \varepsilon=0.7, \mathrm{~N}=2000 \mathrm{r} / \mathrm{min}$. The results showed that thermal and elastic deformation has slight influence on water or seawater lubrication, while they have great influence on the pressure distribution of oil lubrication. As shown in Fig.3, thermal effect has a significant influence on pressure distribution than elastic deformation for oil lubricating. The thermal effect leads to a decrease in the maximum pressure by $40 \%$, going from $34.9 \mathrm{MPa}$ to $21 \mathrm{MPa}$. The elastic deformation leads to a decrease in the maximum pressure by $4.3 \%$, ranging from 34.9 $\mathrm{MPa}$ to $33.4 \mathrm{MPa}$. The maximum temperature of oil film increased by $94.1 \mathrm{~K}$ when taking into account thermal effect, while the maximum temperature decrease by $1 \mathrm{~K}$ when considering the elastic deformation.

\subsection{The influence of different lubricants on the performance of bearings}

The influence of lubricants on the performance of bearings is discussed in this part for $\mathrm{D}=80 \mathrm{~mm}, \mathrm{~L} / \mathrm{D}=1$, $\Phi=0.1 \%, \varepsilon=0.7, \mathrm{~N}=2000 \mathrm{r} / \mathrm{min}$. The lubricants are water, seawater, and lubricating oil, respectively. Film pressure distributions of three different lubricants on the middle ring are shown in Fig.4, and compared with Zhang et al.[10], which used finite difference method with Reynold boundary conditions to validate the model used. 


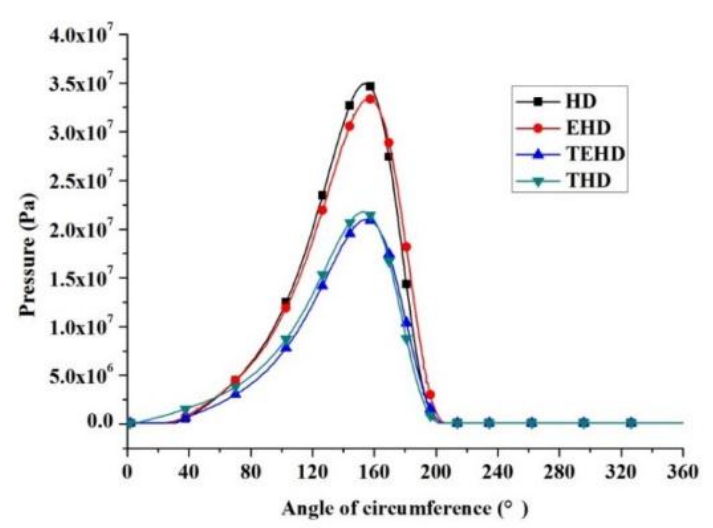

Figure 3. The pressure distribution in the midplane of oil-lubricated journal bearings

For oil lubrication in Fig. 4 a, the pressure from $\theta=0^{\circ}$

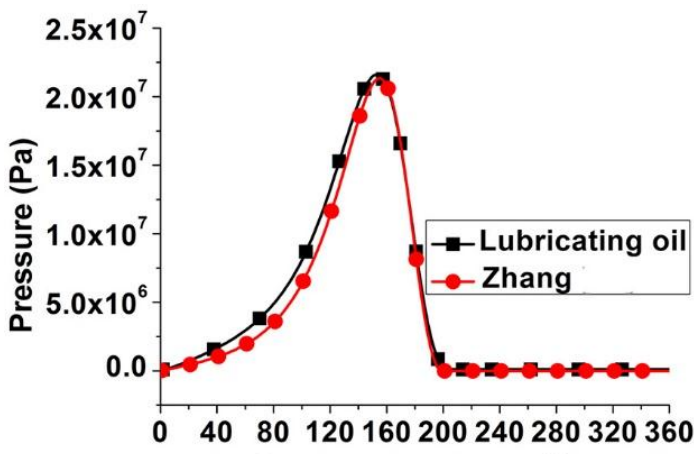

(a) to $153^{\circ}$ calculated in current work is slightly bigger than Zhang et al. [10] for the average viscosity is a bit lower than the actual value when the dimensionless pressure in Zhang et al. [10] is transforming to the actual pressure. From $\theta=153^{\circ}$ to $360^{\circ}$, the pressure distributions for oil lubrication agree well. For water or seawater lubrication in Fig. $4 \mathrm{~b}$, the pressure distributions for $\theta=180^{\circ}$ to $320^{\circ}$ agree well with Zhang et al. [10], while from $\theta=320^{\circ}$ to $360^{\circ}$ the pressure in this work rises from cavitation pressure gradually to a positive value. The positive value leads to a bigger pressure in the beginning of convergent film which explains the difference between this work and Zhang et al. [10] from $\theta=0^{\circ}$ to $180^{\circ}$.

Figure 4. Pressure distribution in circumference

\subsection{Eccentricity ratio effects}
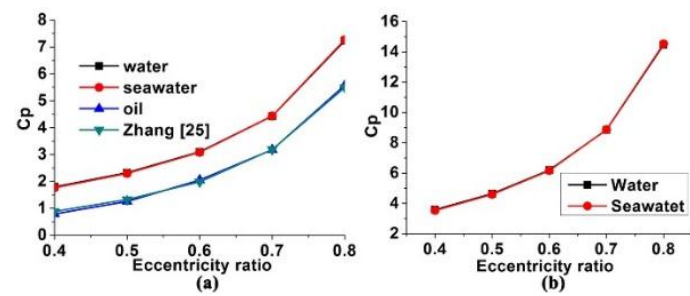

Figure 5. The influence of eccentricity ratio on load carrying capacity of different lubricants
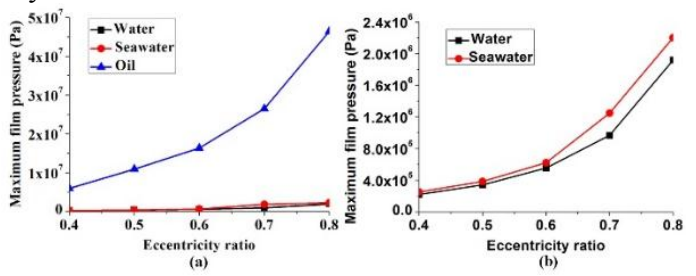

Figure 6. The influence of eccentricity ratio on maximum film pressure
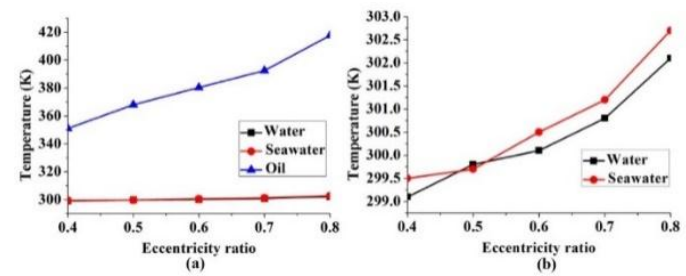

Figure 7. The influence of eccentricity ratio on maximum temperature
The influence of eccentricity ratio on load carrying capacity, maximum temperature, and maximum pressure for different lubricants are shown in Figs.5-7 for $\mathrm{D}=80 \mathrm{~mm}, \mathrm{~L} / \mathrm{D}=1, \Phi=0.1 \%, \mathrm{~N}=2000 \mathrm{r} / \mathrm{min}, \varepsilon=0.4-0.8$. A dimensionless bearing capacity coefficient $C_{p}$ was defined, as shown in equation 13. Fig.5 shows the relationship between bearing capacity coefficient and eccentricity ratio for $\mathrm{L} / \mathrm{D}=1$ and the results in this work are compared with Zhang et al. (1986). The design process can be simplified by the relationship between $C_{p}$ and eccentricity ratio. As $\mathrm{C}_{\mathrm{p}}$ can be calculated by average pressure $\left(p_{m}\right)$, clearance ratio $(\varphi)$, lubricant viscosity $(\eta)$, and rotational speed $(\omega)$, then the eccentricity ratio $(\varepsilon)$ can be determined. Fig. 5 can be used as a reference for the design of bearing by different lubricants, from which eccentricity ratio can be determined according to the capacity coefficient $\mathrm{C}_{\mathrm{p}}$ for different lubricants. The lubrication condition, such as film thickness, can be determined particularly for water lubrication, which is lack of relevant information.

$$
C_{\mathrm{p}}=\frac{2 \mathrm{p}_{\mathrm{m}} \varphi^{2}}{\eta \omega}
$$

The capacity coefficient in Fig.5 goes up with the rise of eccentricity ratio, and the result of oil lubrication agrees well with Zhang et al. (1986). However, for water or seawater lubrication, the result in this work is larger. This is mainly because the film pressure in the divergence region rises from cavitation pressure to a positive value and the positive value leads to a larger pressure in 
convergence region, which result in the capacity coefficient's difference. It reveals the fact that there is a big difference between water and oil lubrication and the theory of oil lubrication is not completely suitable for water lubrication.

The influence of eccentricity ratio on maximum film pressure is shown in Fig.6, which can be used as a reference to estimate the film pressure at different eccentricity ratios. The maximum film pressure has a parabolic increase trend with the moving up of eccentricity ratio for various lubricants. The greater the eccentricity ratio is, the faster the maximum film pressure increases.

The relationship between eccentricity ratio and maximum film temperature is shown in Fig.7. As the eccentricity ratio rises from 0.4 to 0.8 , the maximum temperature increases gradually. The increment of maximum film temperature of oil rises from $348 \mathrm{~K}$ to 417 $\mathrm{K}$, water from $299 \mathrm{~K}$ to $302 \mathrm{~K}$, and seawater from 299.5 $\mathrm{K}$ to $303 \mathrm{~K}$. This is mainly due to the difference of viscosity, specific heat capacity and the thermal conductivity. The temperature rise of oil is so high that it must be considered in numerical calculation, while the temperature rise of water lubrication does not need to be considered as it is less than $4 \mathrm{~K}$.

\subsection{Equivalent substitution of bearings among different lubricants}

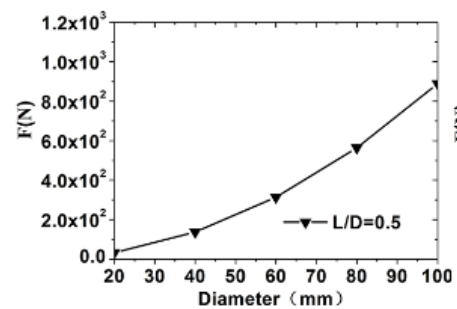

(a) Water lubrication, $\mathrm{L} / \mathrm{D}=0.5$

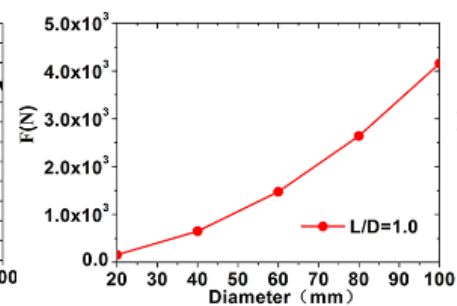

(b) Water lubrication, $\mathrm{L} / \mathrm{D}=1$

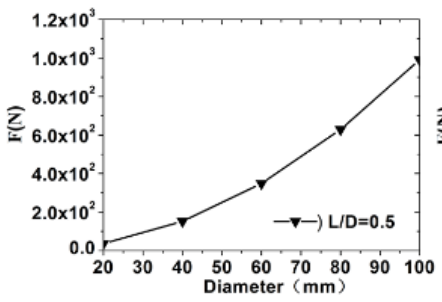

(d) Seawater lubrication, $\mathrm{L} / \mathrm{D}=0.5$

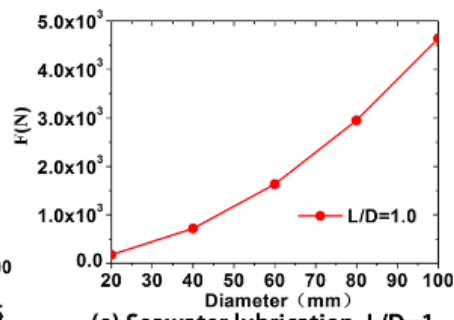

(e) Seawater lubrication, $\mathrm{L} / \mathrm{D}=1$

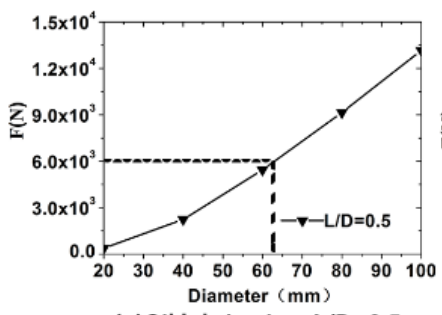

(g)Oil lubrication, $\mathrm{L} / \mathrm{D}=0.5$

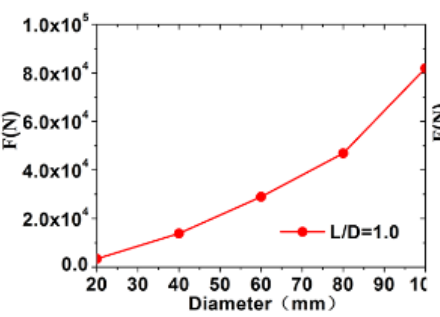

(h) Oil lubrication, $\mathrm{L} / \mathrm{D}=1$
Load carrying capacity comparison of bearings for different lubricants are shown in Fig. 8 for $D=20-100 \mathrm{~mm}$, $\mathrm{L} / \mathrm{D}=0.5-1.5, \Phi=0.1 \%, \varepsilon=0.7, \mathrm{~N}=2000 \mathrm{r} / \mathrm{min}$. To avoid stress concentration caused by high eccentricity ratio, the eccentricity ratio is set to be 0.7 . The $\mathrm{L} / \mathrm{D}$ value is chosen as $0.5-1.5$ for it is a general scope for journal bearings. The load carrying capacity goes up with the increase of bearing diameter, and the trends are similar for different lubricants. Fig. 8 can be used as a reference for the determination of bearing diameters in different L/D especially for water lubrication where the theory of oil reference for the exchange of bearings by different lubricants. When the load carrying capacity is given with he rotational speed being $2000 \mathrm{r} / \mathrm{min}$, the diameter of the journal bearing can be determined by Fig.8. For example, when the required load carrying capacity is $6000 \mathrm{~N}$, the diameter of the water lubricated journal bearing should be $84 \mathrm{~mm}$ with $\mathrm{L} / \mathrm{D}=1.5$, the diameter of the seawater lubrication should be $80 \mathrm{~mm}$ with $\mathrm{L} / \mathrm{D}=1.5$, and the diameter of oil lubrication should be $63 \mathrm{~mm}$ with $\mathrm{L} / \mathrm{D}=0.5$. Thus judgment can be made that hydrodynamic lubrication has been formed by the design in Fig.8.

The present work only presents the effect of $\mathrm{L} / \mathrm{D}$ and diameter on the load carrying capacity of journal bearings in different lubricants. The influences of eccentricity ratio $(\varepsilon)$, relative clearance $(\varphi)$, and rotational speed $(N)$ on the replacement of oil lubrication by water lubrication deserve further study.

Figure 8. Load carrying capacity comparison of bearings with different lubricants

\section{Conclusions}

This study investigates the similarities and differences of plain journal bearings lubricated by water, seawater, and lubricating oil with thermal effect and elastic deformation. The influence of thermal, cavitation and elastic deformation on pressure distribution are studied for 
different lubricants. From the results, the conclusions can be drawn as follows:

1. The maximum temperature of oil-lubricated bearing occurs at the location of minimum film thickness. While for the water and seawater-lubricated journal bearings, the maximum temperature occurs at the location of minimum film thickness and extend $180^{\circ}$ backward.

2. Thermal effect has greater influence on pressure

\section{Acknowledgements}

The authors thank the editor and anonymous reviewers for their helpful comments and suggestions. The work was financially supported by Marine Low Speed Engine Project-Phase I (Grant No. CDGC01-KT11).

\section{References}

1. Y. Q. Wang and C. Li, Advanced Materials Research 299-300, 12 (2011).

2. E. N. Santos, C. J. C. Blanco, E. N. Macêdo, C. E. A. Maneschy, and J. N. N. Quaresma, Tribology International 52, 161 (2012). distribution than elastic deformation for oil lubrication. 3. The capacity coefficient of oil lubrication agrees well with previous work while for water or seawater lubrication the value in this work is larger for the effect of cavitation.

4. A reference for the determination of bearing diameters in different L/D is shown in Fig.8.

3. X. L. Zhang, Z. W. Yin, D. Jiang, and G. Y. Gao, Applied Mechanics \& Materials 711, 27 (2014).

4. G. Gao, Z. Yin, D. Jiang, and X. Zhang, Tribology International 75, 31 (2014).

5. Y. Wang, Z. Yin, H. Li, G. Gao, and X. Zhang, Wear s 380-381, 42 (2017).

6. Y. Wang, Z. Yin, H. Li, and G. Gao, 272, 012022 (2017).

7. X. Zhang, Z. Yin, G. Gao, and Z. Li, Tribology International 85, 37 (2015).

8. W. Litwin and C. Dymarski, Tribology International 95, 449 (2016).

9. W. Litwin, Tribology International 82, 153 (2015).

10. Z. Zhiming, Theory of hydrodynamic lubrication of sliding bearings (Higher Education Pressc, 1986). 